\title{
Disseminated Ulcerated Nodules: A Quiz
}

Zhen-Zhen WANG ${ }^{1}$, Hai-Qin JIANG ${ }^{1 *}$ and Hong-Sheng WANG ${ }^{1,2 *}$

${ }^{1}$ Institute of Dermatology, Chinese Academy of Medical Sciences \& Peking Union Medical College, Jiangsu Key Laboratory of Molecular Biology for Skin Diseases and STIs, and ${ }^{2}$ Center for Global Health, School of Public Health, Nanjing Medical University, Nanjing, China. *E-mail: hq_j1985@126.com; whs33@vip.sina.com

A 68-year-old Asian man presented with multiple painless ulcerated and suppurative pigeon's egg-sized nodules on his neck, left axilla and wrist for 2 years (Fig. 1A-C). He had no fever, dry cough, fatigue, weight loss, or other symptoms. He had emphysema for 10 years and denied a history of tuberculosis. The lesions first appeared on his neck and then spread to the unilateral axilla and wrist. Laboratory examinations indicated that erythrocyte sedimentation rate (ESR) was $70 \mathrm{~mm} / \mathrm{h}$ (normal $<15 \mathrm{~mm} / \mathrm{h}$ ) and C-reactive protein (CRP) was $58 \mathrm{mg} / 1$ (normal $<8.0 \mathrm{mg} / \mathrm{l}$ ). T-SPOT. TB (Oxford Immunotec Ltd, Oxford, UK), an interferon- $\gamma$ release test, indicated positive findings (TB antigen - A $=39$, TB antigen $-\mathrm{B}=8 ;>6$ is positive). Peripheral blood lymphocytes counts and human immunodeficiency virus tests revealed no abnormalities. Computed tomographic (CT) scan revealed left axillary and mediastinal lymphadenopathy with peripheral exudation (Fig 1D).

Histopathology of skin biopsies from a lesion on the patient's neck showed granulomatous inflammation with necrosis and negative results of periodic acid-Schiff, silver and Ziehl-Neelsen stains (Fig. 2). Single PCR tests for rpoB and hsp65 genes of the skin lesions did not identify Mycobacterium tuberculosis until nested PCR for the same genes were performed. Round creamy-yellow colony bacterial colonies were observed after 37 days of culture at $34^{\circ} \mathrm{C}$ in Löwenstein-Jensen medium. Sequence analysis of PCR products of the isolate's $16 \mathrm{~S}$ ribosomal RNA and hsp65 genes also detected M. tuberculosis strain FDAARGOS_756. The patient received the antituberculosis regimen, including rifampin $450 \mathrm{mg} /$ day, isoniazid $300 \mathrm{mg} /$ day, ethambutol 750 $\mathrm{mg} /$ day, pyrazinamide $750 \mathrm{mg}$ /day for 2 months followed by isoniazid $300 \mathrm{mg} /$ day and rifampicin $450 \mathrm{mg} /$ day for 4 months (2HRZE/4HR). After 6 months of follow-up, the lesions were almost completely cleared leaving scars (Fig. $3 \mathrm{~A}-\mathrm{C})$. The enlarged lymph nodes were smaller and surrounding exudation was less than before on CT scan (Fig. 3D). Re-examinations including ESR, CRP and T-SPOT.TB returned to normal with no adverse effects during treatment.

What is your diagnosis? See next page for answer.
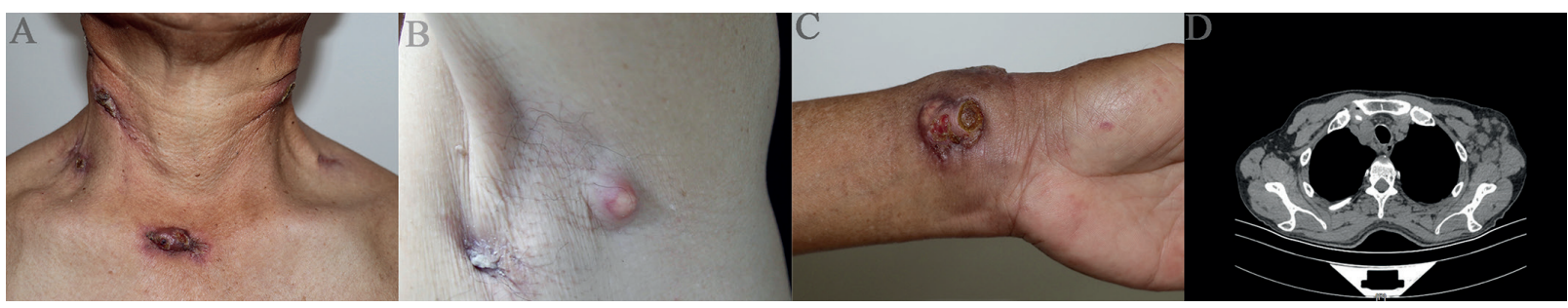

Fig. 1. Clinical photographs and computed tomographic scan before treatment. (A-C) Ulcerated and suppurative pigeon's egg-sized nodules on the bilateral neck, left axilla and wrist of the patient. (D) Lymphadenopathy in left axillary and mediastinal with peripheral exudation.

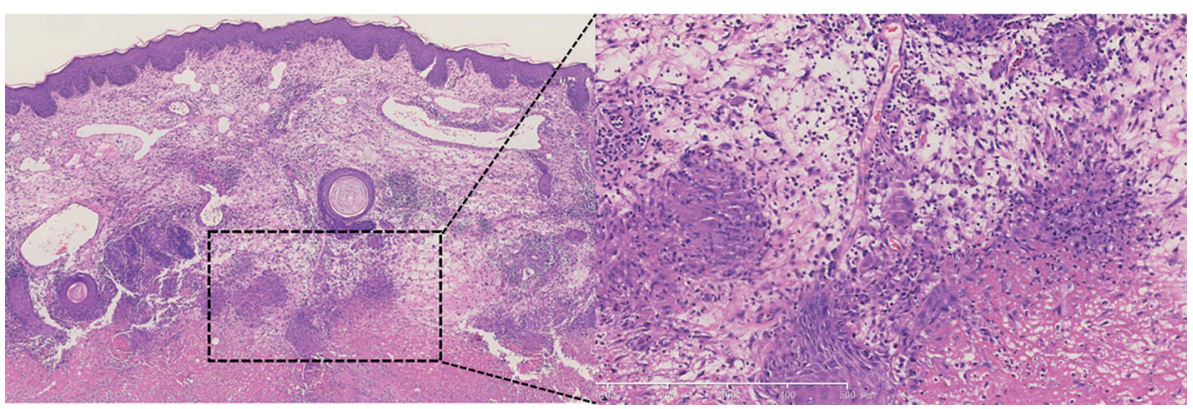

Fig. 2. Infectious granuloma with necrosis and infiltrates of lymphocytes, epithelioid cells and Langhans giant cells. (Haematoxylin and eosin (HE) staining, original magnification: left panel $\times 25$, right panel: $\times 100)$.
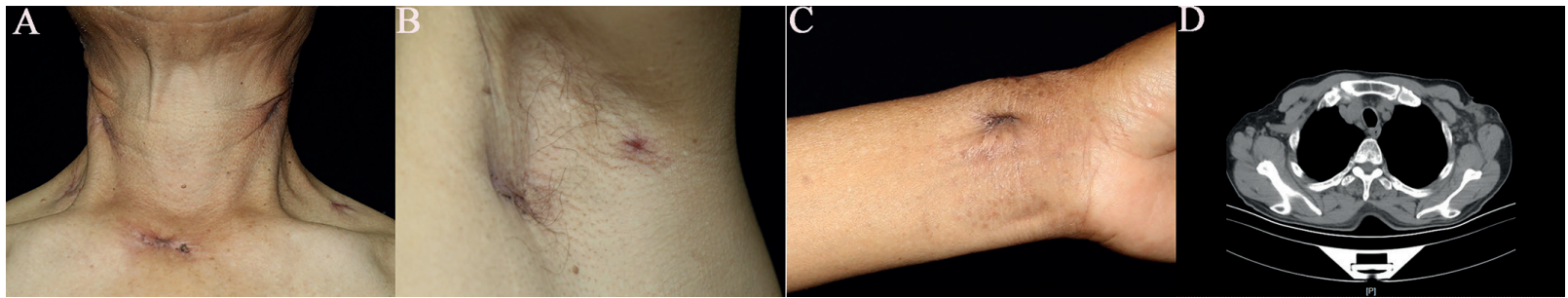

Fig. 3. Clinical photographs and computed tomographic scan after 6 months' treatment. (A-C) The lesions were almost completely cleared leaving scars. (D) Enlarged lymph nodes were smaller and surrounding exudation was reduced. 


\section{ANSWERS TO QUIZ}

\section{Disseminated Ulcerated Nodules: A Commentary}

Acta Derm Venereol 2022; 102: adv00622.

\section{Diagnosis: Disseminated scrofuloderma}

Scrofuloderma is a chronic form of cutaneous tuberculosis (CTB) caused by M. tuberculosis or M. bovis. PCR for RD1 genes (encoding culture filtrate protein 10 and early secretory antigenic target protein 6) of the colony can be performed to distinguish the 2 pathogens (1). The test found no deletion of these 2 virulence-related genes in the pathogen, which meant that the organism was not M. bovis. CTB, which accounts for in $1-2 \%$ of tuberculosis cases, is characterized by diverse clinical features (2). Scrofuloderma may present at any age and usually develops from the direct involvement of the skin overlying a contiguous tuberculosis focus (3). It can result from joint, bone, testis or neck tuberculosis, and it can also uncommonly arise from spermatic cord or scalp tuberculosis $(4,5)$. The typical clinical manifestations of scrofuloderma are asymptomatic dermal and sometimes subcutaneous nodules, and then the lesions gradually expand and break down, leading to ulcers, fistulous tracts and sinus.

Differential diagnoses of scrofuloderma include syphilis, hidradenitis suppurativa, lymphoma, non-tuberculosis mycobacterial infection, deep mycoses, actinomycosis and other infectious disease. A detailed survey of medical history is important. Molecular detection and culture of the skin lesions or the pus aspirated from the lesion are the gold standards of diagnosis to confirm CTB. However, the diagnosis of CTB is challenging and misdiagnoses are not uncommon. Routine laboratory examinations and skin histopathology are not sufficient. Microbiological culture, including fungus, mycobacterium, and bacteria culture, is needed to rule out alternative infectious diseases. Conversely, when the diagnosis of suspected CTB cannot be confirmed by conventional molecular detection, such as conventional PCR, in addition culturing, semi-quantitative PCR using the Xpert MTB/RIF ${ }^{\circledR}$ method or nested and multiplex PCR will be useful $(6,7)$. Interferon- $\gamma$ release test, such as T-SPOT.TB, can also aid in diagnosis due to its high sensitivity and specificity in individuals with tuberculosis (8). CTB can be currently treated with standard multidrug therapy (MDT) like other forms of tuberculosis and be adjusted through culture and susceptibility data (9). Dermatologists should monitor blood count and biochemical parameters regularly, as the treatment requires continuous oral medication for at least 6 months. Attention should be paid to paradoxical reactions, and surgical intervention may sometimes be needed during treatment (10).

Disseminated scrofuloderma reported here is very rare in an immunocompetent patient. This condition can be classified as multifocal systemic tuberculosis because of the presence of 2 or more lesions in extrapulmonary sites (skin; the lymph nodes of neck, axilla and wrist) even without pulmonary involvement. Therefore, once cutaneous tuberculosis is diagnosed, pulmonary and extrapulmonary tuberculosis should be investigated simultaneously. Misdiagnosis of CTB or delay in treatment can make lesions persist and expand, resulting in disfigurement and transmission of infection, and even develop into squamous cell carcinoma. It is necessary to consider the comprehensive medical examinations for suspected CTB patients, especially in atypical cases.

\section{ACKNOWLEDGEMENTS}

The authors thank the patient for permission to use his photographs and clinical data. We also thank Dr Jingshu Xiong, Ying Shi, Wenyue Zhang and Xiaohong Su from the Institute of Dermatology, Chinese Academy of Medical Sciences \& Peking Union Medical College for helping with the pathology and laboratory examinations. This work was supported by the grants from Jiangsu Provincial Science and Technology Project (BE2018619).

The authors have no conflicts of interest to declare.

\section{REFERENCES}

1. Pym AS, Brodin P, Brosch R, Huerre M, Cole ST. Loss of RD1 contributed to the attenuation of the live tuberculosis vaccines Mycobacterium bovis BCG and Mycobacterium microti. Mol Microbiol 2002; 46: 709-717.

2. Franco-Paredes C, Marcos LA, Henao-Martínez AF, RodríguezMorales AJ, Villamil-Gómez WE, Gotuzzo E, et al. Cutaneous mycobacterial infections. Clin Microbiol Rev 2018; 32: e00069-18.

3. Fontanilla JM, Barnes A, von Reyn CF. Current diagnosis and management of peripheral tuberculous lymphadenitis. Clin Infect Dis 2011; 53: 555-562.

4. Jia JA-O, Chen DC, Feng YG. A 9-year massive scrofuloderma of the scalp cured in 3 months. Int J Dermatol 2019; 58: e34-e36.

5. Kumekawa M, Yamamoto T. Cheilitis granulomatosa in a child with juvenile periodontal disease. J Dermatol 2020; 47: e397-e398.

6. Sinha P, Gupta A, Prakash P, Anupurba S, Tripathi R, Srivastava GN. Differentiation of mycobacterium tuberculosis complex from non-tubercular mycobacteria by nested multiplex PCR targeting IS6110, MTP40 and 32kD alpha antigen encoding gene fragments. BMC Infect Dis 2016; 16: 123.

7. Costa LL, Veasey JV. Diagnosis of cutaneous tuberculosis (lymph node scrofuloderma) using the Xpert MTB/RIF(R) method. An Bras Dermatol 2021; 96: 82-84.

8. Chen $Y$, Jiang $H$, Zhang W, Chen Z, Mei Y, Chen H, et al. Diagnostic value of T-SPOT.TB test in cutaneous mycobacterial infections. Acta Derm Venereol 2018; 98: 989-990.

9. van Zyl L, du Plessis J, Viljoen J. Cutaneous tuberculosis overview and current treatment regimens. Tuberculosis (Edinb) 2015; 95: 629-638.

10. Ting KC, Wang GS. Paradoxical reaction in the treatment of scrofula. N Engl J Med 2020; 383: 1767. 